\title{
Determining and Characterizing if Deer Placenta Stem Cells are Present in Commercial Food Supplement Capsules: Utilizing Microscopy, Elemental Analysis, Cytology, Histology, Immunohistochemistry and Flow Cytometry
}

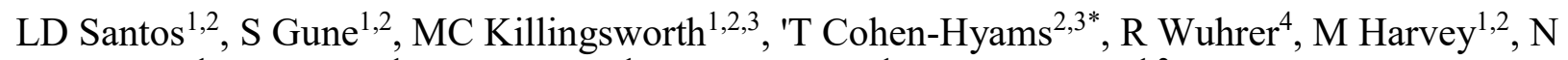

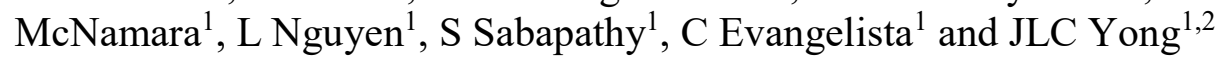

${ }^{1}$ New South Wales Health Pathology, Sydney, Australia.

2. Western Sydney University, Sydney, School of Medicine, NSW, Australia.

${ }^{3 .}$ Ingham Institute for Applied Medical Research, NSW, Australia.

4. Western Sydney University, Sydney, Advanced Materials Characterisation Facility, NSW, Australia.

*Corresponding author: Tzipi.Cohen-Hyams@inghaminstitute.org.au

There are many products on the market claiming the presence of live deer placenta stem cells (SC) in certain food supplement capsules. It has been claimed these live stem cells are able to remain bioactive for 2-4 years, providing live stem cell therapy and therapeutic benefits such as curing or helping to treat many diseases or altering human physiology for better health [1-7]. This research is aimed at determining the presence/absence of live deer placenta stem cells in commercially available capsules.

A prospective study was conducted using eight randomly selected capsules from a total of 420 capsules. The capsules and their contents were documented and characteristics recorded. Examination of the contents was performed using cytology (direct smears, using Papanicolaou and Diff Quick stains and wet mounts with thin and thick preparations), histology, frozen sections (FS) with different thickness and cryostat temperature settings, histochemistry (oil Red O), immunohistochemistry (IHC) and flow cytometry (FC), which used BD FACS Canto II and FACS Diva analysis software. Energy dispersive spectroscopy (EDS) elemental analysis and electron microscopy (EM) was also performed with the latter having two sample preparations: 1) a 3 micron thick cryostat air-dried section mounted on a glass slide for field emission scanning EM, which was carbon coated and examined at $10 \mathrm{kV}$ using the backscattered electron detector, and 2) a glutaraldehyde fixed routinely processed $90 \mathrm{~nm}$ ultrathin section for transmission EM. EM and hemocytometer were used for particle measurement and counting.

Initially optical microscopic investigations were carried out and samples were then prepared for cytology, histology, microanalysis, flow cytometry and electron microscopy. Cytology showed numerous variably-sized oil globules mixed with rhomboidal or needle-like crystalline materials as well as many minute rod-like structures. No SC's were found. Some particles were colorful (Fig 1) ranging from darkly stained to white opaque.

Histology examination revealed variably-sized solid particles, but no SC (Fig 2a-c). IHC showed negative staining with CD31, CD34, CD44, CD45, CD117, CD133, S100 and MNF. With the frozen sections (FS), solid materials were found, but no SC. Oil red O staining showed positive red-staining lipid material of variable size. EDS elemental analysis revealed heterogeneous material that was osmium-rich, suggesting that it contained a high proportion of lipid. Rhomboidal shaped material showed high concentration of uranium suggesting it was rich in proteinaceous substance. No other toxic elements were found apart from those used in the sample processing (Fig 3). Flow Cytometry (FC) revealed the sample was predominantly composed of debris and non-viable material demonstrated by non-specific binding scatterplot patterns. No evidence of stem cells (CD34, CD117, CD45) or major cell 
lineages - myeloid (CD13, CD14, CD15, CD16, CD56, CD33, CD64), T lymphoid (CD2, CD3, CD7), B lymphoid (CD19, CD79b) or rare cell types (CD30, CD103, CD123) were revealed.

Electron microscopy (EM) of the cryostat air-dried section for field emission scanning EM showed lipid, collagen, bacteria and globular and crystalline materials, and the glutaraldehyde section for transmission EM showed bacteria and collagen but no lipid, suggesting the lipid was dissolved away by acetone and ethanol during EM processing. No SC's were found (Fig 3e and f). The size of the oil globules ranged from 50 to 500 microns (um). The size of the solid particles measured up to 1,000 um.

There were no powdery or cake-like materials which are the usual appearances of freeze-dried substances. Within the contents, oil globules, collagen, bacteria, protein, lipid and many other solid, crystalline materials were observed. There were no live SC found through the techniques. The study could be improved by studying the deer placenta SC features and comparing to the supplement. If SC were present, they would unlikely be able to enter the circulation intact and alive. SC would be broken down by the gastrointestinal system and its enzymes. The large size of mesenchymal mammalian SC does not differ much between mouse (average, 28 um) and elephant (average, 29 um) and most likely will not pass through the microvilli of enterocytes.

\section{References:}

[1] https://purtier.com

[2] http://riway.com/sg/en/product/jsp

[3] https://purtierplacenta.ph/

[4] https://purtierway.com/purtier-placenta

[5] https://www.purtierstemcellph.com/purtierplacenta

[6] https://www.lazada.com.my/purtier-placenta-stem-sell-therapy-riwa-60-capsules/

[7] https://www.purtierworld.com/traditional-stem-cell-therapy-vs-purtier-placenta-live=-cell-therapy
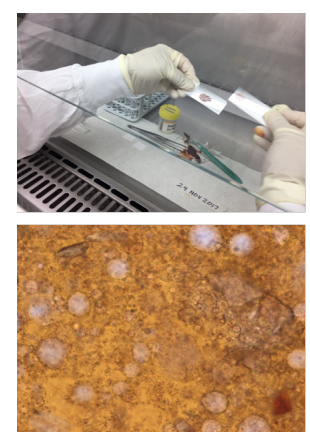

Figure 1. Cytology.
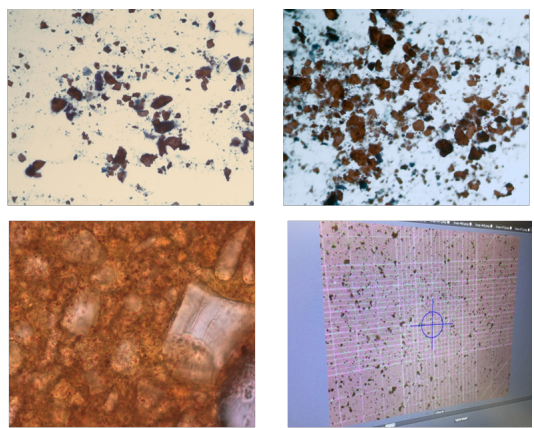

Papanicolaou x 100, c) Diff Quick x 100. d) We Mount $\times 100$, e) Wet Mount $\times \quad 400,-f$ Hemocytometer (with a count of $0.053 \times 109 / \mathrm{L}$ solid particles determined).

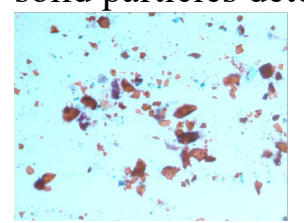

a)

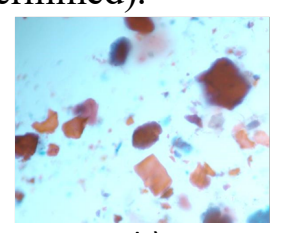

b)

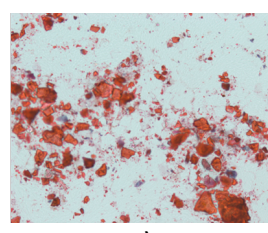

c)
Figure 2. a) and b) Histology H\&E x100 \& x400 and c) showing the frozen section $\mathrm{x} 100$.

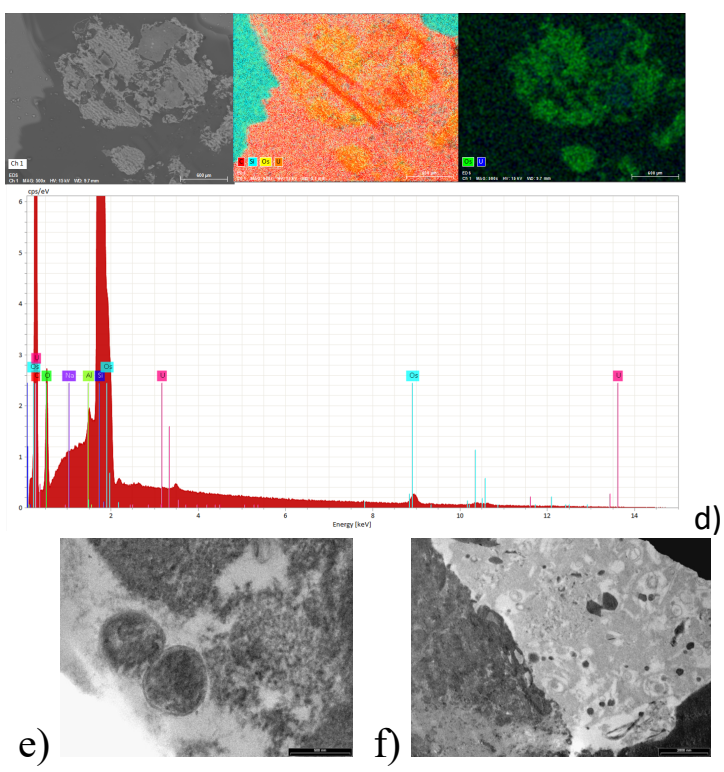

Figure 3. a) to c) SEM/EDS analysis showing osmium-rich globular components in yellow (b) or green (c) and uranium-rich crystalline material in dark blue (c). Corresponding spectral peaks are shown below (d). TEM showing e) bacteria and f) crystalline and globular material. 